\title{
High Rates of Readmission in Necrotizing Pancreatitis: Natural History or Opportunity for Improvement? Short Title: Readmission in Pancreatitis
}

Thomas K. Maatman ${ }^{1 *}$, Sarakshi Mahajan ${ }^{1 *}$, Alexandra M. Roch ${ }^{1}$, Kyle A. Lewellen ${ }^{2}$, Mark A. Heimberger ${ }^{2}$, Cameron L. Colgate ${ }^{3}$, Eugene P. Ceppa ${ }^{1}$, Michael G. House ${ }^{1}$, Attila Nakeeb ${ }^{1}$, C. Max Schmidt ${ }^{1}$, Nicholas J. Zyromski ${ }^{1}$

${ }^{1}$ Department of Surgery, Indiana University School of Medicine, Indianapolis, IN, USA

${ }^{2}$ Indiana University School of Medicine, Indianapolis, IN, USA

${ }^{3}$ Center for Outcomes Research in Surgery (CORES), Indiana University School of Medicine, Indianapolis, IN, USA

*Drs. Maatman and Mahajan contributed equally to this project and should be considered coprimary authors.

\section{Correspondence:}

Nicholas J. Zyromski, MD

Professor of Surgery

Department of Surgery, Indiana University School of Medicine

545 Barnhill Dr., EH 519

Indianapolis, IN 46202

Office: (317) 944-5012; Fax: (317) 274-4554; Email: nzyromsk@iupui.edu

$\dagger$ This work has been presented in part as a poster presentation at the $59^{\text {th }}$ annual DDW-SSAT meeting and the $51^{\text {st }}$ annual meeting of The Pancreas Club in Washington D.C. (2018)

Author Contribution:

Drs. Maatman and Mahajan were involved in acquisition, analysis, and interpretation of data in addition to conception, drafting and revising the work. Dr. Zyromski serves as the mentor and corresponding author for the work; he was involved in the conception, interpretation, and revision of the work, final approval, and agreement to be accountable for the work. Drs. Ceppa, House, Schmidt, and Nakeeb were involved in the revision and final approval of the version to be published. Dr. Roch, K. Lewellen, and M. Heimberger assisted in analysis of data and revision of the work. C. Colgate performed the statistical analysis of the data.

There was no financial support or grant assistance utilized in the production of this study.

This is the author's manuscript of the article published in final edited form as:

Maatman T, Mahajan S, Roch A, Lewellen K, Heimberger M, Colgate C, Ceppa E, House M, Nakeeb A, Schmidt C, Zyromski N. High Rates of Readmission in Necrotizing Pancreatitis: Natural History or Opportunity for Improvement? J Gastrointest Surg. 
Background:

Necrotizing pancreatitis (NP) is a complex and heterogeneous disease with a protracted disease course. Hospital readmission is extremely common; however, few data exist regarding cause of readmission in NP.

Methods:

A retrospective review of NP patients treated between 2005-2017 identified patients readmitted both locally and to our hospital. All patients with unplanned hospital readmissions were evaluated to determine cause for readmission. Clinical and demographic factors of all patients were recorded. As appropriate, two independent groups t-tests and Pearson's correlation or Fisher's exact tests were performed to analyze the relationship between index admission clinical factors and readmission. P-values of $<0.05$ were accepted as statistically significant.

Results:

601 NP patients were reviewed. Median age was 52 years (13-96). Median index admission length of stay was 19 days (2-176). The most common etiology was biliary (49.9\%) followed by alcohol (20.0\%). Unplanned readmission occurred in 432 patients (72\%) accounting for a total of 971 unique readmissions (mean readmissions/patient - 2.3). The most common readmission indications were symptomatic necrosis requiring supportive care and/or intervention $(31.2 \%)$, infected necrosis requiring antibiotics and/or intervention (26.6\%), failure to thrive (9.7\%), and non-necrosis infection (6.6\%). Patients rering readmission had increased incidence of index admission renal failure $(21.3 \%$ vs. $14.2 \%, \mathrm{p}=0.05)$ and cardiovascular failure $(12.5 \% \mathrm{vs} .4 .7 \%$, $\mathrm{p}=0.01)$.

Discussion:

Readmission in NP is extremely common. Significant portions of readmissions are a result of the disease natural history; however, a percentage of readmissions appear to be preventable. Patients with organ failure are at increased risk for unplanned readmission and will benefit from close follow-up.

Key words: necrotizing pancreatitis, readmission, risk factors, organ failure 


\section{$\underline{\text { Introduction }}$}

Acute pancreatitis (AP) is an increasingly common indication for hospital admission and is associated with a significant financial burden to the health care system; this cost is estimated at over \$2.2 billion annually(1). Most patients experience mild AP and recover uneventfully following a short hospital stay with little to no long-term sequelae. Patients with mild AP do not typically require readmission to the hospital following index admission. In contrast, severe acute pancreatitis (SAP) with pancreatic necrosis develops in $10-20 \%$ of all $\mathrm{AP}(1,2)$ and is characterized by a prolonged hospital stay. These patients frequently require hospital readmission. Additionally, long-term sequelae in this population is much more common and often requires intervention.

Necrotizing pancreatitis (NP) is a complex disease and carries significant morbidity with mortality ranging from $15-30 \%(3,4)$. Morbidity includes organ failure, infectious complications, need for procedural intervention, malnutrition, and physical deconditioning. The result of this devastating disease process is an extremely high rate of hospital readmission and a prolonged recuperation.

Readmission rate is an increasingly utilized metric of patient care quality (5). Given the cost burden readmission represents to the health care system, there is an ever-increasing interest in predicting and preventing hospital readmission to improve patient care efficiency and quality. In all patients with AP, readmission is seen in about 1 of every 5 patients. The more severe the sentinel episode, the more likely the risk of readmission (6-8). However, little is known about the incidence and etiology of readmission specifically in NP patients, the most vulnerable group. 
We hypothesized that specific causes for readmission are identifiable and may allow the opportunity to treat patients preemptively, avoiding the need for unplanned hospital readmission. Therefore, the aim of this study is to evaluate the incidence and etiology of readmission following index admission with necrotizing pancreatitis and identify risk factors for readmission.

\section{Materials and Methods}

The institutional necrotizing pancreatitis database was reviewed to identify NP patients treated at Indiana University Health University Hospital (IU-UH) between 2005 and 2017. This database contains demographic and clinic information of 647 NP patients treated at IU-UH during that time. All patients were included regardless of etiology, age, or treatment strategy. Patients were excluded from review if they did not survive index admission or were lost to follow-up.

Acute pancreatitis and severe acute pancreatitis were defined according to the revised Atlanta classification(9). Necrosis was identified as a lack of pancreatic parenchymal enhancement and/or findings of peripancreatic necrosis such as acute necrotic collection (ANC) or walled off necrosis (WON) on contrast enhanced cross sectional imaging(9). Dedicated faculty pancreatic radiologists confirmed necrosis in all cases. Index admission data include: age, sex, etiology, comorbidities, presence of organ failure (as defined according to the Modified Marshall scoring system for organ dysfunction $(9,10))$, presence of infected necrosis, and need for intervention.

Treatment during the patient's index admission is largely supportive care and avoids intervention of pancreatic necrosis, if possible. Treatment strategy follows the consensus 
guidelines as published by the International Association of Pancreatology and the American Pancreatic Association(11).

Electronic medical records were queried for readmissions to IU-UH and to all hospitals in which electronic medical records are shared. All readmissions were captured in those patients included in the final analysis. This was accomplished via electronic medical records or through patient records at follow-up visits, as all patients are followed until disease resolution.

Readmission dates, indication for readmission, and interventions were recorded. The time period for readmission was individualized to each patient - readmissions were included if they occurred prior to the individual patient's disease resolution. Unplanned readmissions during the active disease were of interest. Planned readmissions were not included in the study and were defined as any readmission scheduled for planned intervention (percutaneous, endoscopic, or surgical). Additionally, readmissions resulting from long-term sequelae were not included in the analysis. Therefore, disease duration was recorded and all readmissions following resolution of NP were excluded from analysis.

Two-tailed Student's t-tests and Pearson's correlations or Fisher's exact tests were performed, as appropriate, to analyze the relationships between readmission and suspected risk factors identified during the index admission. P-values of $<0.05$ were accepted as statistically significant. Those factors with $\mathrm{p}$-value $>0.10$ in univariate analysis were included in a multivariate analysis. All data were compiled and recorded in strict compliance with the protocols and guidelines set forth by the Institutional Review Board (IRB), which approved the conduct of this study.

\section{$\underline{\text { Results }}$}




\section{Patient characteristics}

A total of 647 NP patients were treated between 2005 and 2017; 77\% (501/647) of patients were transferred to our facility and the remaining $23 \%(146 / 647)$ were admitted primarily. Regional distribution of the NP patient population treated at IU-UH is show in Figure 1. Median age at onset was 52 years (13-96) and $65.5 \%$ of patients were male. The most common etiology was biliary $(49.6 \%)$, followed by alcohol $(20.2 \%)$, hypertriglyceridemia (6.0\%), post-ERCP (5.7\%), and drug-induced (2.0\%). Index admission mortality was 5.0\% (32 patients) and a total of 14 patients (2.2\%) were lost to follow-up; $601 \mathrm{NP}$ patients were therefore included in the analysis. The median time to disease resolution was 5 months (1-73) and median follow-up was 27 months (1-160). Overall mortality from necrotizing pancreatitis was $8.8 \%$.

\section{Readmission}

At least one disease related unplanned readmission was seen in 432 patients (72\%). The total number of unique unplanned readmissions was 971; each patient was readmitted an average of 2.3 times $(+/-0.1$, standard error). The most common indications for readmission were: infected necrosis requiring either antibiotic therapy or intervention $(17.8 \%)$, symptomatic necrosis requiring supportive care $(13.0 \%)$, symptomatic necrosis requiring intervention $(11.0 \%)$, and failure to thrive (6.0\%) (Figure 2). Etiologies of all unplanned readmissions are detailed in Table 1.

\section{Prediction of Readmission}

No pre-existing comorbidity was found to increase a patient's risk for readmission (COPD, asthma, coronary artery disease, congestive heart failure, hypertension, diabetes, tobacco use, or obesity). The presence of clinical factors of interest during index admission in 
each group is shown in Table 2. There was no significant difference in mean age, etiology, median index admission length of stay (LOS), infected necrosis, or respiratory failure in patients requiring readmission when compared to patients without readmission. Patients requiring necrosis intervention during the index hospital admission $(n=169,28.1 \%)$ had no difference in readmission rate when compared to those without intervention $(69.8 \%$ vs. $75.0 \%, p=0.92)$. Patients requiring readmission were more likely to have developed renal or cardiovascular failure during the index hospital admission. The likelihood of readmission increased significantly with progressive organ failure (Figure 3).

\section{$\underline{\text { Discussion }}$}

This retrospective analysis defines incidence, etiology, and risk factors for readmission following index admission in a large group of necrotizing pancreatitis patients treated at a highvolume pancreatic quaternary referral center. Readmission following an episode of NP is extremely common (72\%); much higher than that seen in other hepatopancreatobiliary pathology(12). Importantly, this study lends insight into reasons underlying readmission, which offers opportunity to improve clinical practice.

Previous studies report rates of readmission in acute pancreatitis ranging from $19-34 \%$ (8, 13). Moreover, these studies identified risk factors associated with readmission: male sex, alcohol etiology, admission to an intensive care unit, inpatient length of stay greater than 7 days, gastrointestinal symptoms at discharge, discharge on less than a solid diet, and discharge with drains in place $(8,13)$. Additionally, Whitlock et al developed a scoring system at discharge to predict 30-day readmission in all patients with acute pancreatitis - less than solid diet (3 points), GI symptoms ( 3 points), pancreatic necrosis ( 2 points), antibiotics ( 2 points), pain ( 1 point). Patients with a score of four or greater had between a $68-87 \%$ chance of 30 -day hospital 
readmission(14). Multiple risk factors and a high readmission risk score are present in nearly every NP patient, and therefore do not necessarily provide the additional clinical information required to identify those patients at highest risk for readmission among the NP population.

Our findings are in accordance with previous studies that have shown increased severity of disease to correlate with increased readmission risk(6-8). However, etiology of readmission in NP has not been described by previous studies. A major finding of our current analysis is that one-third of readmissions are due to symptomatic necrosis and an additional one-quarter are due to infected necrosis. Thus, a large proportion of readmissions in NP are related to the natural history of the disease and as such, may not be preventable. In contrast, we identified several etiologies for readmission that may offer the ability to decrease readmission. These etiologies include: failure to thrive, non-necrosis infection, and tube dysfunction. Collectively, this group accounts for $21 \%$ of readmissions; initiatives to decrease readmission rates in NP should therefore target these problems.

Necrotizing pancreatitis is a complex and heterogeneous problem best treated with a multidisciplinary approach and advanced techniques most often found at a specialty center. While treatment at regional referral centers provides full access to advanced treatment strategies, regionalization adds complexity to the follow-up care of each patient. The geographic distribution of our NP patient population is shown in Figure 1. The vast majority of our NP patients live more than 50 miles away; this distance adds challenge to continuing NP patient care through the several months long disease process.

This study highlights an opportunity to coordinate the management of some NPassociated problems to patient's local services such as primary care physicians and community emergency rooms and hospitals. These problems include: symptomatic necrosis requiring 
supportive care, failure to thrive, non-necrosis infection, and tube dysfunction - in total accounting for $48 \%$ of readmissions identified in the current analysis. Obviously, coordinated communication between dedicated caregivers at the local and regional treatment centers is critical to this shared care model. More complex problems will continue to require management at specialty centers, particularly symptomatic necrosis requiring intervention, infected necrosis, disconnected pancreatic duct syndrome, gastrointestinal fistula, pancreaticobiliary stricture, and hemorrhage. This paradigm of shared regional and specialty center care mandates excellent communication between physicians.

The results of this study have sparked several important changes in our necrotizing pancreatitis practice. Patients at high-risk for readmission, particularly those who developed organ failure during their index admission, are targeted for close follow-up (ideally, one to two weeks following discharge). Additionally, a dedicated pancreatitis nurse coordinator has been tasked with frequent communication with high-risk patients (one to two times per week) to identify developing deviations from expected recovery. Patients with more straightforward problems, as able, are managed at local facilities with frequent communication between the local physician and responsible hepatopancreatobiliary surgeon or medical pancreatologists. The combination of early follow-up and frequent communication with patients will ideally improve the ability to plan admissions when intervention is required.

Strategies aimed to decrease hospital readmission are often focused on post-discharge care. It may be possible to improve treatment strategies in these patients during the initial hospital admission which can decrease unplanned readmission. However, it appears that no particular treatment or necrosis intervention during the index admission effects readmission rate. This highlights the fact that currently no treatment exists to modulate the disease course in NP; 
treatment is strictly supportive and reactive. Additionally, no consensus discharge criteria exist in NP patients. The development of standardized discharge criteria in this population is the scope of future projects. Unplanned readmission rates may improve with discharge criteria individualized to the necrotizing pancreatitis patient population.

This study is limited by is retrospective nature - chart review may not completely capture the patient's clinical picture at the time of readmission. Due to the complexity of necrotizing pancreatitis, many patients are likely readmitted with a combination of pathology, and not one specific problem resulting in readmission. Nevertheless, thorough analysis of these data has identified several clearly consistent categories of readmission etiology. An important strength of this study is its high-volume nature and thorough long-term statewide follow up, both of which are difficult to obtain in this extremely complicated patient population.

\section{Conclusion}

The complex disease process of necrotizing pancreatitis results in an extremely high rate of hospital readmission $-72 \%$. A portion of hospital readmissions are unavoidable as they are inherent to this long-term disease. All necrotizing pancreatitis patients should be considered high-risk for readmission; however, this analysis has identified patients with increasing numbers of organ failure to be the highest-risk population for readmission. Close follow-up and frequent communication with local primary care providers are strategies to decrease readmission in this vulnerable population.

\section{$\underline{\text { References }}$}


1. Fagenholz P, Fernández-del Castillo C, Harris N, Pelletier A, Camargo Jr. C. Direct Medical Costs of Acute Pancreatitis Hospitalizations in the United States. Pancreas. 2007;35(4):302-7.

2. Howard TJ, Patel JB, Zyromski N, Sandrasegaran K, Yu J, Nakeeb A, Pitt HA, Lillemoe KD. Declining morbidity and mortality rates in the surgical management of pancreatic necrosis. $J$ Gastrointest Surg. 2007;11(1):43-9.

3. Kokosis G, Perez A, Pappas TN. Surgical Management of Necrotizing Pancreatitis: An Overview. World J Gastroenterol. 2014;20(43):16106-12.

4. Freeman M, Werner J, van Santvoort H, Baron T, Besselink M, Windsor J, Horvath K, vanSonnenberg E, Bollen T, Vege S. Interventions for Necrotizing pancreatitis: Summary of a Multidisciplinary Consensus Conference. Pancreas. 2012;41(8):1176-94.

5. Fischer C, Lingsma HF, Marang-van de Mheen PJ, Kringos DS, Klazinga NS, Steyerberg EW. Is the Readmission Rate a Valid Quality Indicator? A Review of the Evidence. PLOS One. 2014;9(11):e112282.

6. Yadav D, Lee E, Papchristou G, O'Connell M. A Population-Based Evaluation of Readmissions After First Hospitalization for Acute Pancreatitis. Pancreas. 2014;43(4):630-7.

7. Yadav D, O'Connell M, Papachristou GI. Natural history following the first attack of acute pancreatitis. Am J Gastroenterol. 2012;107(7):1096-103.

8. Vipperla K, Papachristou GI, Easler J, Muddana V, Slivka A, Whitcomb DC, Yadav D. Risk of and factors associated with readmission after a sentinel attack of acute pancreatitis. Clin Gastroenterol Hepatol. 2014;12(11):1911-9.

9. Banks PA, Bollen TL, Dervenis C, Gooszen HG, Johnson CD, Sarr MG, Tsiotos GG, Vege SS, Acute Pancreatitis Classification Working G. Classification of acute pancreatitis-2012: revision of the Atlanta classification and definitions by international consensus. Gut. 2013;62(1):102-11.

10. Marshall J, Cook D, Christou N, Bernard G, Sprung C, Sibbald W. Multiple Organ Dysfunction Score: A Reliable Descriptor of a Complex Clinical Outcome. Critical Care Medicine. 1995;23(10):1638-52.

11. Working Group IAPAPAAPG. IAP/APA evidence-based guidelines for the management of acute pancreatitis. Pancreatology. 2013;13(4 Suppl 2):e1-15.

12. Idrees J, Rosinksi B, Merath K, Chen Q, Bagante F, Pawlik TM. Readmission after pancreatic resection: causes, costs, and cost-effectiveness analysis of high versus low quality hospitals using the Nationwide Readmission Database. HPB (Oxford).

2018; https://doi.org/10.1016/j.hpb.2018.07.011.

13. Whitlock TL, Repas K, Tignor A, Conwell D, Singh V, Banks PA, Wu BU. Early readmission in acute pancreatitis: incidence and risk factors. Am J Gastroenterol. 2010;105(11):2492-7.

14. Whitlock TL, Tignor A, Webster EM, Repas K, Conwell D, Banks PA, Wu BU. A scoring system to predict readmission of patients with acute pancreatitis to the hospital within thirty days of discharge. Clin Gastroenterol Hepatol. 2011;9(2):175-80. 
Figure 1: Distribution of necrotizing pancreatitis patients treated at Indiana University Health University Hospital between 2005 and 2017. The Indiana University (IU) logo represents the location of our University Hospital.

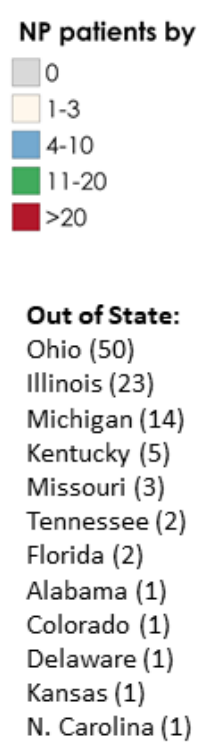

Created with mapchart.net $\odot$

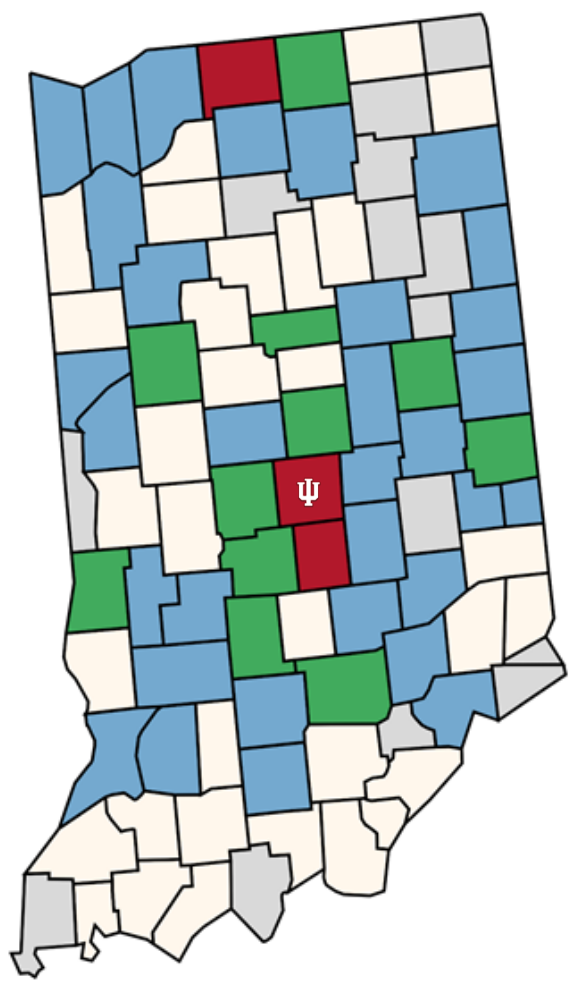


Figure 2:Readmission in necrotizing pancreatitis and the most common etiologies

\section{5-2016}

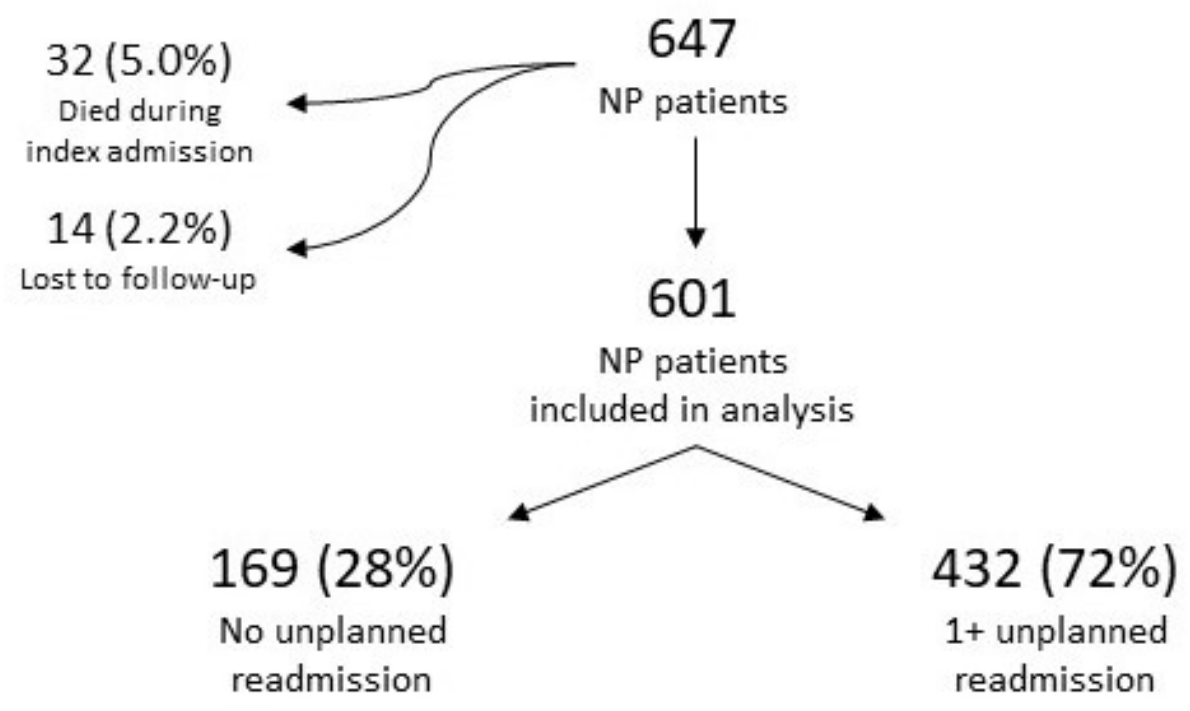

971 unique readmission events Average readmissions/patient: 2.3 (+/-0.1)

Most common etiologies:

- Infected necrosis req. antibiotics or intervention-258/971(26.666)

- Symptomatic necrosis req. supportive care-167/971(17.256)

- Symptomatic necrosis req. intervention-136/971(14.096)

- Failure to thrive $-94 / 971$ (9.796) 
Table 1: Indication for unplanned readmission by diagnosis (total number of unplanned readmissions $=$ 971). Abbreviations: CLABSI-central-line associated blood stream infection.

\begin{tabular}{|c|c|}
\hline Admission indication & n (\%) \\
\hline Symptomatic necrosis & $303(31.2 \%)$ \\
\hline Supportive care & $167(17.2 \%)$ \\
\hline Percutaneous drain & $10(1.0 \%)$ \\
\hline Endoscopic therapy & $24(2.5 \%)$ \\
\hline Operative therapy & $102(10.5 \%)$ \\
\hline Infected necrosis & $258(26.6 \%)$ \\
\hline Antibiotics & $30(3.1 \%)$ \\
\hline Percutaneous drain & $109(11.2 \%)$ \\
\hline Endoscopic therapy & $6(0.6 \%)$ \\
\hline Operative therapy & $113(11.6 \%)$ \\
\hline Failure to thrive & $94(9.7 \%)$ \\
\hline Infection, non-necrosis & $64(6.6 \%)$ \\
\hline CLABSI & $22(2.3 \%)$ \\
\hline Pneumonia & $17(1.8 \%)$ \\
\hline Urinary tract infection & $10(1.0 \%)$ \\
\hline C. difficile & $9(0.9 \%)$ \\
\hline Cholangitis & $6(0.6 \%)$ \\
\hline Gastrointestinal fistula & $46(4.7 \%)$ \\
\hline Tube dysfunction & $46(4.7 \%)$ \\
\hline Percutaneous drain & $33(3.4 \%)$ \\
\hline Feeding tube & $13(1.3 \%)$ \\
\hline Disconnected pancreatic duct syndrome & $38(3.9 \%)$ \\
\hline Pancreatico-biliary stricture & $30(3.1 \%)$ \\
\hline Biliary & $20(2.1 \%)$ \\
\hline Duodenal & $7(0.7 \%)$ \\
\hline Pancreatic duct & $3(0.3 \%)$ \\
\hline Hemorrhage & $35(3.6 \%)$ \\
\hline Pseudoaneurysm & $17(1.8 \%)$ \\
\hline Percutaneous drain & $11(1.1 \%)$ \\
\hline Gastrointestinal & $7(0.7 \%)$ \\
\hline Venous thromboembolism & $12(1.2 \%)$ \\
\hline Bowel obstruction/perforation/ischemia & $9(0.9 \%)$ \\
\hline Wound complication & $7(0.7 \%)$ \\
\hline Recurrent acute pancreatitis & $4(0.4 \%)$ \\
\hline Cardiac events & $4(0.4 \%)$ \\
\hline Other/unknown & $28(2.9 \%)$ \\
\hline
\end{tabular}


Table 2: Clinical factors of interest and comparison between groups (readmission vs. no readmission). Bolded values represent statistical significance with p-value $<0.05$. Abbreviations: LOS - index admission length of stay

\begin{tabular}{|l|l|l|l|}
\hline Variable & Readmission & No readmission & \multicolumn{2}{l|}{-value } \\
\hline Age, years (mean +/- SD) & $52.5+/-15.3$ & $49.9+/-15.5$ & 0.06 \\
\hline Median LOS, days & 19 & 20 & 0.17 \\
\hline Infected necrosis (\%) & $19.9 \%$ & $19.5 \%$ & 0.92 \\
\hline Intervention - index admission (\%) & $25.0 \%$ & $30.2 \%$ & 0.92 \\
\hline Respiratory failure (\%) & $30.6 \%$ & $25.4 \%$ & 0.22 \\
\hline Renal failure (\%) & $21.3 \%$ & $14.2 \%$ & $\mathbf{0 . 0 5}$ \\
\hline Cardiovascular failure (\%) & $12.5 \%$ & $4.7 \%$ & $\mathbf{0 . 0 1}$ \\
\hline Number of organ failure & & & $\mathbf{0 . 0 2}$ \\
0 & $61.6 \%$ & $69.2 \%$ & \\
1 & $17.4 \%$ & $17.2 \%$ & \\
2 & $12.3 \%$ & $9.5 \%$ & \\
3 & $8.8 \%$ & $4.1 \%$ & \\
\hline
\end{tabular}


Figure 3: Readmission risk increases with progressive organ failure. This trend of increasing readmission risk is statistically significant, $p=0.02$.

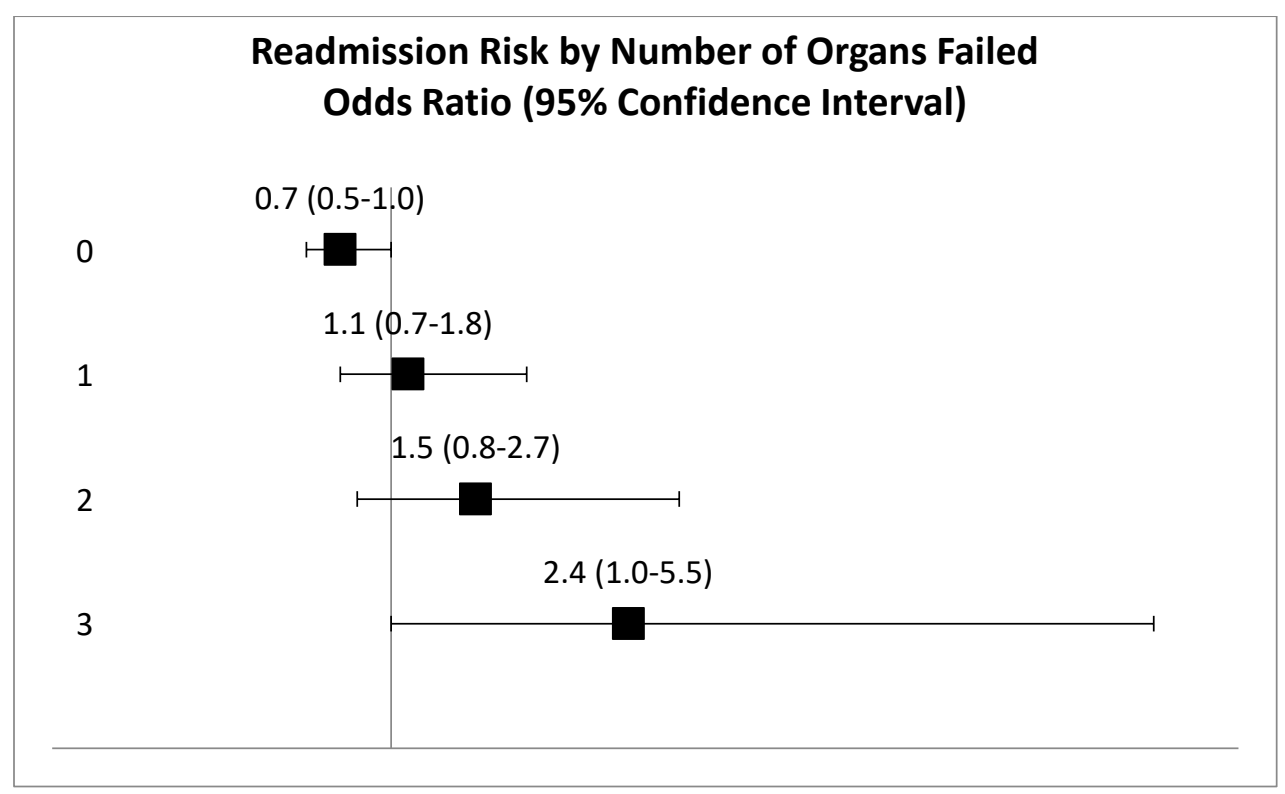

